\title{
Comparison of siRNA-induced off-target RNA and protein effects
}

\author{
LOURDES M. ALEMÁN, ${ }^{1,2}$ JOHN DOENCH, ${ }^{3}$ and PHILLIP A. SHARP ${ }^{1,2}$ \\ ${ }^{1}$ Center for Cancer Research, Massachusetts Institute of Technology, Cambridge, Massachusetts 02139, USA \\ ${ }^{2}$ Department of Biology, Massachusetts Institute of Technology, Cambridge, Massachusetts 02139, USA \\ ${ }^{3}$ Department of Biological Chemistry and Molecular Pharmacology, Harvard Medical School, Boston, Massachusetts 02115, USA
}

\begin{abstract}
The downregulation of many mRNAs has been observed through bioinformatic analysis of microarray results following transfection of short interfering RNAs (siRNAs). Many of these mRNA changes are due to the interaction of the siRNA guide strand with partially complementary sites and thus are considered "off-target" effects. To examine the mRNA:siRNA interactions important for off-target effects, we generated a panel of mRNA:siRNA combinations containing single and double mismatches, bulges, and noncanonical base-pairing interactions in the 9th, 10th, and 11th positions of two siRNA binding sites located in the $3^{\prime}$ UTR of an integrated reporter gene. Approximately half of the mRNA:siRNA combinations containing mismatches in positions 9-11 result in a twofold or more mRNA decrease with varying degrees of protein knockdown. However, mRNA and protein analysis of the various mRNA:siRNA combinations reveals instances in which mRNA and protein levels do not correlate. Analysis of the resulting degradation products recovered from an imperfectly complementary siRNA interaction with an endogenous gene reveals a small fraction of products that map to the canonical siRNA cleavage site. Furthermore, downregulation of ARGONAUTE 2 (AGO2), the only AGO family protein known to catalyze canonical siRNAmediated cleavage, did not significantly affect the degree of mRNA knockdown observed for one of the stably expressed reporters after transfection of an imperfectly complementary siRNA. These results indicate that although some degree of canonical siRNA cleavage can take place between a siRNA and an off-target transcript, most off-target mRNA reductions are likely attributable to AGO2-independent degradation processes.
\end{abstract}

Keywords: siRNA; off-target effects; G:U wobble; siRNA canonical cleavage; AGO2

\section{INTRODUCTION}

Short interfering RNAs (siRNAs) have emerged as a powerful experimental tool to study gene function, given their ability to cause downregulation of target messages in a sequence-specific manner. Perfectly complementary siRNAs mediate sequence-specific silencing by inducing mRNA cleavage and subsequent mRNA degradation of target transcripts. siRNAs guide the RNA-induced silencing complex (RISC) to mediate endonucleolytic cleavage of the target mRNA at the site opposite the 10th and 11th positions of the guide siRNA strand (Martinez et al. 2002). Within the RISC complex, cleavage is catalyzed by a specific member of the ARGONAUTE (AGO) protein family (Liu

\footnotetext{
Reprint requests to: Phillip A. Sharp, Center for Cancer Research, Massachusetts Institute of Technology, Cambridge, MA 02139, USA; e-mail: sharppa@mit.edu; fax: (617) 253-3867.

Article published online ahead of print. Article and publication date are at http://www.rnajournal.org/cgi/doi/10.1261/rna.352507.
}

et al. 2004; Song et al. 2004). Although all four mammalian AGO proteins are capable of binding both siRNAs and microRNAs (miRNAs), only AGO2 is able to perform RNA cleavage in mammals (Liu et al. 2004).

siRNA-mediated mRNA downregulation was initially reported to be highly specific (Chi et al. 2003; Semizarov et al. 2003). Additionally, several reports demonstrated that even single nucleotide mismatches between the siRNA strand and the target mRNA greatly decreased the rate of target mRNA cleavage (Ding et al. 2003; Haley and Zamore 2004; Martinez and Tuschl 2004). However, microarray analyses have shown that siRNAs with only partial complementarity to mRNAs can also cause a reduction in the RNA levels of a large number of transcripts (Jackson et al. 2003, 2006). This type of mRNA regulation has been termed "off-target" effects and is typically associated with less than twofold mRNA downregulation (Jackson et al. 2003, 2006; Saxena et al. 2003; Persengiev et al. 2004; Scacheri et al. 2004; Holen et al. 2005; Lin et al. 2005; Birmingham et al. 2006; Schwarz et al. 2006). Further 
analysis of the off-target mRNAs revealed that this mRNA effect is determined by complementarity of the mRNA to the $5^{\prime}$ region of the guide siRNA strand. This type of complementarity is reminiscent of canonical miRNA regulation, in which the same 5 ' region plays a central role in mRNA:miRNA interactions (Brennecke et al. 2003, 2005; Doench and Sharp 2004; Kloosterman et al. 2004). This region, specifically nucleotides $2-7$ at the 5 ' end of the miRNA strand, is considered the "seed region" (Lewis et al. 2005). mRNA complementarity to the $3^{\prime}$ region of a miRNA is not generally required for miRNA function, but it is likely that additional complementarity to this region results in enhanced miRNA-mediated downregulation.

A recent series of microarray experiments observed changes in the mRNA levels of a large number of transcripts in HeLa cells after transfection of siRNAs corresponding to two tissue-specific miRNAs, miR-1 in muscle cells and miR-124 in neuronal cells (Lim et al. 2005). A high percentage of the downregulated transcripts $(88 \%$ for miR-1 and $76 \%$ for miR-124) had complementarity to the seed region of the corresponding miRNA. These results indicate that the interaction between the seed region of a partially complementary siRNA guide strand and a particular mRNA could be similar to the interaction observed between a miRNA and its target. Further analysis revealed that compared to all other cell types, the specific tissue in which the miRNA was exclusively expressed also exhibited the lowest expression of the downregulated transcripts. This biological correlation indicates that the downregulated transcripts identified by this microarray study are most likely endogenous targets of miR-1 and miR-124. Thus, in this case the "off-target" interactions between the miR-1 and miR-124 siRNAs and their corresponding downregulated mRNAs probably reflects the physiological interaction between an miRNA and its target mRNA population.

The finding that partially complementary siRNAs can cause reductions in mRNA levels suggests that mammalian miRNAs could regulate their targets by decreasing mRNA levels, in addition to attenuating translation. Support for this hypothesis has been provided by several recent studies, which independently reported that miRNAs can reduce the mRNA levels of partially complementary target genes (Bagga et al. 2005; Jing et al. 2005; Lim et al. 2005; Wu and Belasco 2005; Behm-Ansmant et al. 2006; Giraldez et al. 2006; Rehwinkel et al. 2006; Schmitter et al. 2006; $\mathrm{Wu}$ et al. 2006). Zebrafish miR-430, for example, has been shown to downregulate hundreds of maternal mRNAs during early embryonic development (Giraldez et al. 2006). Furthermore, two miRNAs that had been previously reported to act primarily by translational repression, Caenorhabditis elegans lin-4 and let-7, also reduce the levels of their target transcripts, lin-14 and lin-28 (Bagga et al. 2005). How miRNAs mediate the downregulation of mRNA targets is not well understood. It is known that at least some miRNAs direct mRNA degradation through deadenylation and subsequent degradation of target transcripts (Behm-Ansmant et al. 2006; Giraldez et al. 2006; $\mathrm{Wu}$ et al. 2006). This process probably involves processing bodies (P bodies), which are cytoplasmic sites of mRNA degradation (Jakymiw et al. 2005; Liu et al. 2005; Sen and Blau 2005). In addition, there is evidence that at least one miRNA, miR-16, mediates the rapid destruction of certain AU-rich element (ARE) containing mRNAs by targeting these transcripts for ARE-mediated mRNA degradation (Jing et al. 2005). Given the similarities between off-target mRNA downregulation and miRNA-mediated RNA degradation, it is likely that both of these effects share common mechanisms.

Previous experiments in our laboratory suggested that siRNAs designed to function like miRNAs induced strong protein reduction with little change in mRNA levels (Doench et al. 2003). In these experiments, HeLa cells were transiently transfected with a siRNA that bound to four or six partially complementary binding sites in the 3' UTR of a luciferase reporter construct. Using the same siRNAs and targets, we subsequently observed a twofold decrease in mRNA levels when the mRNAs were expressed from an integrated gene (data not shown). Under these conditions, the silencing at the protein level was 30-fold. Here we address the question of whether interactions between partially complementary siRNA guide strands and mRNAs produce tightly correlated changes in mRNA degradation and translational efficacy. We have concentrated on dissecting the biological significance of positions 9-11 in off-target gene regulation, given the importance of the siRNA central region in mRNA cleavage (Haley and Zamore 2004). To this end, we have introduced single base substitutions at positions 9-11 in stably expressed reporters containing two identical siRNA binding sites and used different siRNAs to create various mRNA:siRNA combinations. Quantitative RNA and protein analysis was performed in parallel for each combination to measure the degree of silencing at the RNA and protein levels. To probe the potential mechanisms involved in offtarget mRNA downregulation, degradation products (corresponding to the 3' UTR of an endogenous gene) generated by partially complementary siRNAs have been mapped, and the importance of AGO2 in this process has been assessed.

Here we report that various mRNA:siRNAs combinations result in different levels of mRNA reduction. We observe instances in which the extent of mRNA and protein knockdown do not correlate, underscoring the importance of assessing siRNA-mediated effects by measuring both mRNA and protein levels. Furthermore, isolation and sequencing of degradation products identify a variety of products; some are likely the result of a canonical siRNA cleavage event while others are probably generated by AGO2-independent degradation processes. 


\section{RESULTS}

\section{Importance of complementarity within positions 9-11 for off-target effects}

Off-target mRNA downregulation could be due to endonucleolytic cleavage reminiscent of canonical siRNA cleavage. When a siRNA is perfectly complementary to its target site, canonical siRNA cleavage occurs between the 10th and 11th nucleotides opposite the siRNA guide strand. Therefore, we decided to test whether complementarity within this region is important for off-target mRNA downregulation. Single mismatches in this region of complementarity are known to dramatically reduce mRNA cleavage in vitro (Haley and Zamore 2004; Martinez and Tuschl 2004; Schwarz et al. 2006). We designed six 3' UTR constructs containing two CXCR4 binding sites with single base substitutions in either the 9th, 10th, or 11th positions. Each CXCR4 binding site is base-paired at position 8 and contains an adenosine in position 1 opposite the siRNA guide strand. Both of these features were associated with the strongest degree of mRNA downregulation for miR-1 and miR-124 targets (C. Nielsen, N. Shomron, R. Sandberg, E. Hornstein, J. Kitzman, C. Burge, unpubl.). Thus, this sequence configuration allows for maximal mRNA knockdown in our studies. Two binding sites were used instead of one to increase the strength of potential siRNA-mediated effects. For each construct, the two identical binding sites were cloned into the $3^{\prime}$ UTR of the reporter Renilla luciferase gene. Five different siRNAs were used to generate a total of 30 mRNA:siRNA combinations: 28 mRNA:siRNA combinations containing single and double mismatches at the 9th, 10th, and/or 11th positions and two perfectly complementary mRNA:siRNA combinations (Table 1).

The Renilla luciferase constructs containing variations of the CXCR4 binding site in their 3' UTRs were transfected into HeLa cells and stable cell lines were established. These cell lines were transfected with $5 \mathrm{nM}$ of siRNA. This concentration was chosen after titration experiments with a stably expressed firefly luciferase reporter construct containing six binding sites showed that the resulting twofold decrease in mRNA levels did not vary significantly with increasing siRNA concentrations (data not shown). RNA and protein levels were measured at $48 \mathrm{~h}$ posttransfection. This duration was selected because both maximal RNA and protein knockdown are observed at $48 \mathrm{~h}$. Time course experiments performed with two of the five siRNAs utilized in this study, CXCR4 and CXCR4-B (Table 1), indicated that mRNA downregulation peaks $24 \mathrm{~h}$ after siRNA transfection and remains the same $48 \mathrm{~h}$ posttransfection, whereas protein knockdown peaked $48 \mathrm{~h}$ after siRNA treatment (data not shown). RNA and protein analyses were performed by quantitative real-time PCR and luciferase assays, respectively. Both of these assays have the advantage of being highly quantitative, allowing the
TABLE 1. RNA and protein knockdown for 30 mRNA:siRNA combinations

$$
\begin{aligned}
& \text { CXCR4 wildtype mRNA GUUUUCAC UCC AGCUAACA } \\
& \begin{array}{c}
\text { CXCRA sirNA } \\
\text { (antisense) }
\end{array}
\end{aligned}
$$

GCC CCC UAC UUC UCA UCU

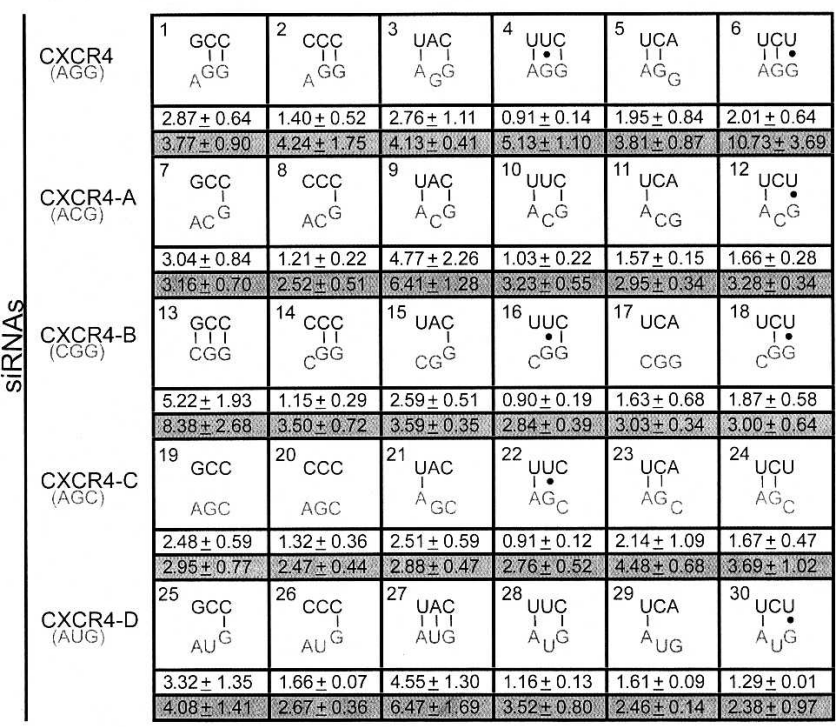

$\square=$ Normalized RNA knockdown (fold change \pm S.D.)

= Normalized protein knockdown (fold change \pm S.D.)

detection of even small changes in both RNA and protein. For each mRNA:siRNA combination, RNA and protein measurements were made from at least five independent experiments and the average results are reported.

Changes in the 9th, 10th, and 11th positions had varied effects on both mRNA and protein levels (Fig. 1; Table 1). In all cases, the decrease in luciferase activity was greater than the decrease in mRNA levels. This suggested that the reduction in protein was the result of a combination of mRNA downregulation and translational repression (Fig. 1A). Perfectly complementary combinations 13 and 27 resulted in an average $4.9 \pm 1.2$-fold mRNA decrease and an average $7.5 \pm 1.6$-fold protein knockdown (Fig. 1A; Table 1). Reductions in mRNA and protein levels greater than these are probably limited by the fraction of cells successfully transfected.

The average RNA and protein decreases for all the mRNA:siRNA combinations were $2.1 \pm 0.1$-fold and $4.0 \pm 0.2$-fold, respectively. Notably, $43 \%$ of the combinations containing mismatches between the 9th and the 11th positions resulted in at least a twofold mRNA decrease (Fig. 1A). Many of these downregulated mRNA:siRNA combinations contained both single and double mismatches in the 9-11 region. This degree of mRNA 
A

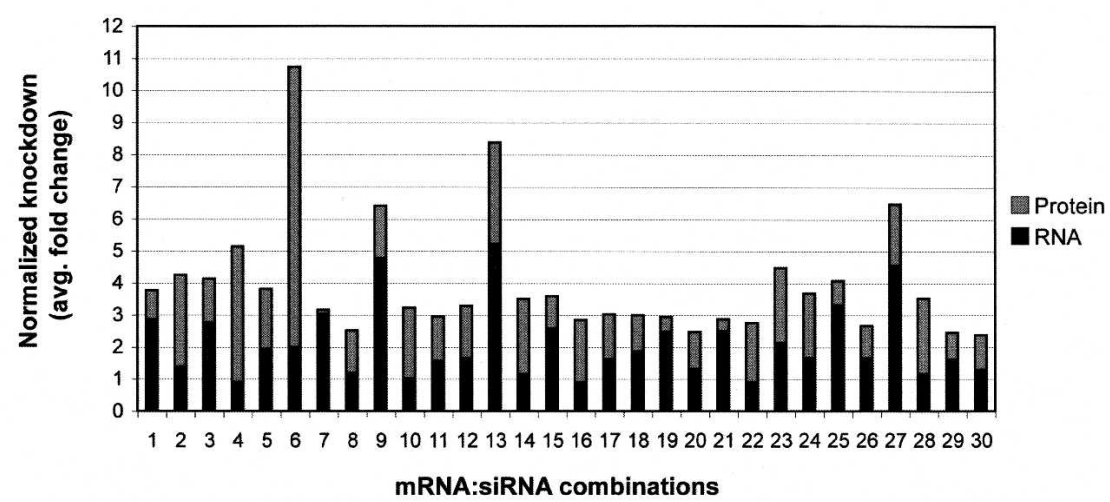

B

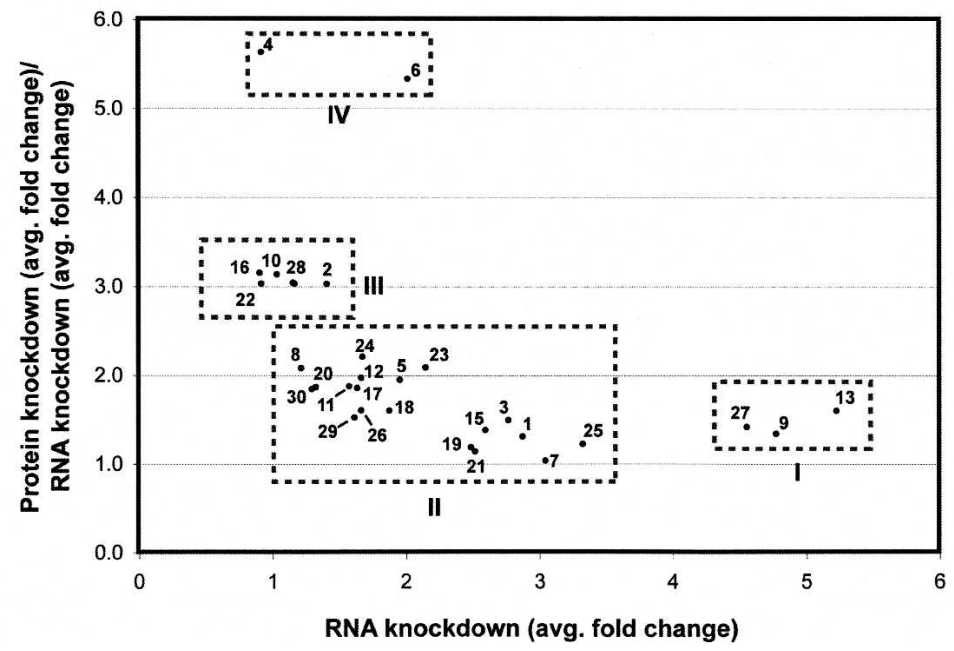

FIGURE 1. Effects of mismatches in positions 9-11 on RNA and protein levels. (A) Average normalized RNA and protein reductions, expressed as fold change, of the 30 mRNA:siRNA combinations (Table 1). Five CXCR4 siRNAs (Table 1), each containing a single nucleotide change within positions 9-11, were transfected into six different stable cell lines expressing Renilla luciferase reporters, which also contained a single nucleotide change within positions 9-11 in both CXCR4 binding sites. Cells were collected for RNA and protein analysis $48 \mathrm{~h}$ after transfection. RNA analysis was performed by quantitative real-time PCR. TBP was used as an internal control. Protein analysis was performed by Renilla luciferase assays. For both RNA and protein analysis, samples were normalized to an unrelated siRNA (targeting GFP) transfection. For each mRNA:siRNA combination the average normalized RNA and protein reductions (fold change) were calculated from at least three independent experiments. Each bar graph represents the average normalized RNA reduction (black) overlapped with the average normalized protein reduction (gray), both expressed as fold change, for a particular mRNA:siRNA combination. Each combination is numbered and represented in Table 1. (B) Ratio of normalized protein knockdown to normalized RNA knockdown (fold change) for each of the 30 mRNA:siRNA combinations. The normalized protein reduction (expressed as fold change) of each mRNA:siRNA combination was divided by its corresponding normalized RNA reduction (fold change) to calculate the ratio between these two measurements. The protein to RNA ratio was then plotted versus normalized RNA knockdown (fold change) for each combination. The combinations were grouped into four distinct subgroups (labeled I-IV) according to their distribution in this plot.

knockdown paralleled the twofold mRNA reduction that we previously observed between the imperfectly complementary CXCR4 siRNA and the firefly reporter construct containing six CXCR4 binding sites (Petersen et al. 2006).
Several important observations emerged from RNA and protein analysis of the 30 different mRNA:siRNA combinations. First, changes at the protein level do not always correlate with changes at the RNA level. In some cases, there were large changes in protein levels with small changes in mRNA levels (Fig. 1A; Table 1). Subgroups of responses resulting from different combinations are apparent when the results are plotted as the normalized average mRNA knockdown versus the ratio of normalized protein knockdown to normalized RNA knockdown (with all values expressed as fold change) (Fig. 1B). One subgroup contains mRNA:siRNA combinations that are significantly regulated at the mRNA level with similar changes at the protein level (Fig. 1B, subgroup I-combinations 9, 13, and 27). As expected this subgroup largely consists of perfectly complementary combinations (Table 1, combinations 13 and 27). A second subgroup of mRNA:siRNA combinations caused intermediate, two- to threefold changes at both mRNA and protein levels (Fig. $1 \mathrm{~B}$, subgroup II-combinations $1,3,5$, $7,8,11,12,15,17-21,23-26,29$, and $30)$. These first two subgroups demonstrate protein changes that closely parallel changes at the mRNA level. A third subgroup is composed of a series of combinations that produced moderate protein downregulation despite very little mRNA knockdown (Fig. 1B, subgroup III-combinations $2,10,16,22$, and 28). Finally, the last subgroup includes only two combinations (4 and 6 ), both of which caused a large degree of protein knockdown and exhibited little to no change in mRNA levels. One possible explanation for the small change in RNA observed for these combinations at $48 \mathrm{~h}$ is that at this time point the RNA could be recovering from repression while the protein levels still reflect the repressed state. This alternative explanation is unlikely given that the siRNA that gave rise to combinations 4 and 6, CXCR4 siRNA, was also utilized for the time point experiments described previously. These experiments established that Renilla luciferase mRNA repression begins to recover after the $48 \mathrm{~h}$ time point (data not shown). In 
addition, Renilla luciferase's protein half-life is $\sim 5.3 \mathrm{~h}$ (Bronstein et al. 1994). It is unlikely that the mRNA recovered substantially from siRNA repression with no detectable recovery at the protein level. Therefore, the third and fourth subgroups likely reflect combinations for which protein knockdown is largely due to translational repression.

RNA and protein analysis showed that different types of mismatches resulted in different degrees of protein knockdown. Among all nucleotide changes, single $\mathrm{G}: \mathrm{U}$ mismatches at position 9 or 10 correlated with the highest levels of translational repression (Table 1, combinations 4 and 6). For example, a single G:U mismatch at the 10th position (Table 1, combination 4) resulted in protein knockdown $(5.13 \pm 1.10$-fold $)$ comparable to that of perfectly complementary mRNA:siRNA combinations (Table 1, combinations 13 and 27). This particular G:U mismatch, however, failed to cause mRNA degradation. The G: $U$ mismatch in the 9th position, combination 6 , showed higher protein knockdown (10.7 \pm 3.7 -fold) in comparison to perfect complementary mRNA:siRNA combinations 13 and 27 (Fig. 1A; Table 1). The mRNA levels for this combination decreased only twofold. Unlike single G:U mismatches, double nucleotide changes containing both a G:U mismatch and a second mismatch resulted in protein knockdown equivalent to that observed for double nucleotide changes lacking a G:U mismatch (Table 1). The average protein knockdown for these mRNA:siRNA combinations was $2.9 \pm 0.3$-fold. For combinations containing two mismatches other than a $\mathrm{G}: \mathrm{U}$, the average protein knockdown was $3.0 \pm 0.2$-fold.

We also considered whether the type of nucleotide at positions 9-11 in the mRNA affected the degree of mRNA knockdown. We observed an average mRNA knockdown of $2.6 \pm 0.1$-fold for mRNA reporter constructs containing a combination of purine and pyrimidine nucleotides at positions 9-11 (all combinations contained in the first, third, and fifth columns of Table 1). In contrast, mRNA reporter constructs containing only pyrimidine nucleotides in positions 9-11 caused a smaller, $1.3 \pm 0.1$-fold mRNA decrease (all combinations contained in the second, fourth, and sixth columns of Table 1). This analysis excluded perfectly complementary mRNA:siRNA combinations 13 and 27. However, the average protein knockdown for reporter mRNAs containing either purines and pyrimidines at positions 9-11 or pyrimidines only was $3.7 \pm 0.2$-fold and $3.7 \pm 0.3$-fold, respectively. Therefore, for these combinations, the nucleotide composition of positions 9-11 affected the degree of mRNA knockdown, but not the degree of protein knockdown. These results suggest that mRNA suppression may be greater if a purine base is present in the 9-11 region in mismatched mRNA:siRNA interactions, while translational repression might not be affected in these circumstances.

\section{Imperfectly complementary siRNA binding yields canonical cleavage products}

We observed mRNA decreases even when mismatches were created at positions 10 and 11, the same positions that are engaged by canonical siRNA-mediated cleavage and that are important for the efficiency of this process (Haley and Zamore 2004). To determine if decreases in mRNA caused by imperfectly complementary siRNAs could result from endonucleolytic cleavage within the siRNA binding site, we mapped 3 '-terminal degradation intermediates after transfection with two siRNAs that bound with imperfect complementarity to the endogenous CXCR4 gene. These experiments focused on an endogenous gene to permit mapping of degradation products relative to a single siRNA binding site on a mRNA. One siRNA, CXCR4-A, resulted in a mismatch at position 10 . The other siRNA, CXCR4-B, resulted in a mismatch at position 11 (Fig. 2A). In addition, three control siRNAs were used: one with perfect complementarity to the endogenous CXCR4 gene and two unrelated siRNAs against GFP and firefly luciferase, respectively. Mapping of degradation products was performed using rapid amplification of cDNA ends ( $5^{\prime}$ RACE) on mRNA from HeLa cells $48 \mathrm{~h}$ after siRNA transfection. Canonical siRNA-mediated cleavage at position 10 results in a $3^{\prime}$ cleavage product that contains a $5^{\prime}$ phosphate and can therefore act as a substrate for $5^{\prime}$ RACE (Kasschau et al. 2003). Various reports have shown that the $3^{\prime}$ cleavage product is degraded by the $5^{\prime}-3^{\prime}$ exonuclease XRN1 and that $3^{\prime}$ fragments accumulate when XRN1 is suppressed (Gazzani et al. 2004; Souret et al. 2004; Orban and Izaurralde 2005). To stabilize the $3^{\prime}$ cleavage products resulting from direct siRNA targeting in our experiments, XRN1 was concomitantly knocked down by RNAi.

5' RACE analysis of RNA from cells transfected with the previously mentioned siRNAs revealed bands of $\sim 250$ base pairs, which is consistent with the size of the expected amplified cleavage product and the location of the primers used during nested PCR (data not shown). In total, 171 amplified products were sequenced and analyzed; $43 \%$ (73/171) of the degradation products mapped to the siRNA binding site, while $57 \%$ (98/171) of the degradation products mapped to regions upstream and downstream of the siRNA binding site (Fig. 2B,C). Notably, 93\% (68/73) of the products that mapped to sequences spanning the siRNA target site were recovered from cells transfected with either perfectly or imperfectly complementary CXCR4 siRNAs. In contrast, only 7\% (5/73) of the products that mapped to sequences spanning the siRNA target site were recovered from cells transfected with control siRNAs. These results indicate that the cleavage products corresponding to the siRNA-targeted region were likely generated by the specific binding of perfectly and imperfectly complementary CXCR4 siRNAs. Significantly, 47\% (34/73) of the 
A CXCR4 Endogenous mRNA $\underset{\text { (antisense) }}{\mathrm{CXCR4} \text { siRNA }}$

GUUUUCAC UCC AGCUAACA

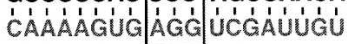

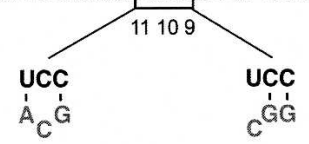

SIRNA CXCR4-A

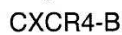

B

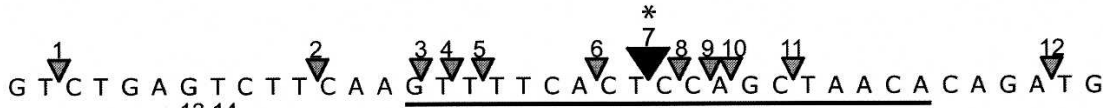
TA A A A G

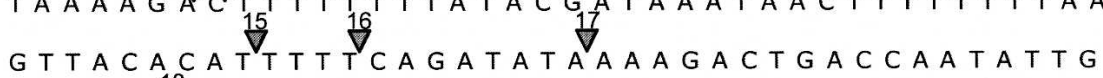
TA C A G T T T T A T T G C T T G T T G G A T T T T T G T C T T G T T T T T C

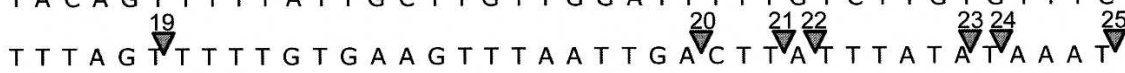
TTTTTTTGTTTCATATTGATGTGTGTCTAGGCAGG

SiRNA Target

CXCR4 inner primer

C

\begin{tabular}{|c|c|c|c|c|c|c|c|c|c|c|c|c|c|c|c|c|c|c|c|c|c|c|c|c|c|}
\hline \multirow[b]{2}{*}{ Cleavage Product } & & \multicolumn{10}{|c|}{ * } & \multirow[b]{2}{*}{12} & \multirow{2}{*}{\multicolumn{2}{|c|}{\begin{tabular}{|l|l|}
13 & 14 \\
\end{tabular}}} & \multirow{2}{*}{15} & \multirow{2}{*}{\multicolumn{2}{|c|}{\begin{tabular}{l|l} 
& \\
16 & 1
\end{tabular}}} & \multirow{2}{*}{\begin{tabular}{|l|l|} 
& \\
& 18 \\
\end{tabular}} & \multicolumn{2}{|c|}{, } & \\
\hline & 1 & 2 & 3 & 4. & 5 & 6 & 7 & 8 & & 10 & 11 & & & & & & & & 19 & $20 \mid 2$ & 21 & 22 & 23 & 24 & 25 \\
\hline $\operatorname{GFP}(30)$ & & & 4 & & 1 & & & & & & & & 24 & & & & & & & & & & 1 & & \\
\hline Firefly luciferase (30) & & & & & & & & & & & & & & & & & & & 3 & & 1 & 2 & & 6 & 18 \\
\hline CXCR4 (44) & & & & & & & 20 & 2 & 10 & 9 & & 2 & & & 1 & & & & & & & & & & \\
\hline CXCR4-A (39) & 2 & 2 & & 4 & & & 10 & & 2 & & & 11 & & & & 6 & & & & 1 & & & & 1 & \\
\hline \begin{tabular}{|l|} 
CXCR4-B (28) \\
\end{tabular} & & & & 1) & & & 4 & & 4 & & 1 & 6 & 7 & 1 & & & & 2 & & & & & & & \\
\hline
\end{tabular}

FIGURE 2. Imperfectly complementary siRNAs result in degradation products that correspond to canonical siRNA-mediated cleavage events. (A) Schematic of the interaction between two CXCR4 siRNAs that contain nucleotide changes in position 10 (CXCR4-A) and position 11 (CXCR4-B) and the endogenous CXCR4 transcript. The perfectly complementary CXCR4 siRNA (antisense strand) is also shown binding to the endogenous CXCR4 mRNA for reference. (B) 5' RACE analysis performed on the endogenous CXCR4 mRNA after transfection with imperfectly complementary siRNAs. HeLa cells were transfected with $100 \mathrm{nM}$ of Xrn1 siRNAs and $10 \mathrm{nM}$ of either control siRNA (GFP), perfectly complementary CXCR4 siRNA, or imperfectly complementary siRNA (CXCR4-A or CXCR4-B). Cells were collected 48 $\mathrm{h}$ after siRNA transfection and subjected to RNA ligation followed by reverse transcription, nested PCR, and TOPO cloning. Each number corresponds to a distinct degradation product. The canonical siRNA cleavage product is highlighted with an asterisk. The CXCR4 siRNAs target sequence and the CXCR4 inner reverse PCR primer are indicated. $(C)$ Summary of the 5' RACE analysis performed on the endogenous CXCR4 mRNA after transfection with perfectly and imperfectly complementary siRNAs. The total number of clones sequenced and the number of clones that mapped to each degradation site are indicated for all the transfected siRNAs. Degradation products that mapped to the siRNA binding site have been highlighted.

products that mapped to the sequence spanning the siRNA target site corresponded to the canonical cleavage site (10th position) and these sequences were only recovered from cells transfected with CXCR4 siRNAs. As expected, the perfectly complementary CXCR4 siRNA yielded degradation products that corresponded to the canonical cleavage site. Forty-five percent $(20 / 44)$ of the degradation products recovered from cells transfected with the perfectly complementary CXCR4 siRNA mapped to the canonical cleavage site. This percentage is similar to prior results reported for analysis of siRNA-directed cleavage (Schmitter et al. 2006).

As previously noted, sequence analysis of amplified products resulting from treatment with the imperfectly complementary siRNAs CXCR4-A and CXCR4-B revealed a low percentage of cleavage products that corresponded to the canonical siRNA cleavage site (Fig. 2C). Twenty-six percent (10/39) of the products recovered from cells transfected with the CXCR4-A siRNA mapped to the canonical cleavage site, whereas $14 \%(4 / 28)$ of the products recovered from cells transfected with the CXCR4-B siRNA mapped to the canonical cleavage site. None of the degradation products recovered from cells transfected with the control siRNAs mapped to the canonical siRNA cleavage site. This observation suggests that the degradation products that were cloned from cells transfected with imperfectly complementary siRNAs and whose sequences mapped to the 10th position are likely the result of canonical siRNA-directed cleavage events.

\section{Some off-target mRNA changes probably occur via AGO2- independent degradation pathways}

As previously mentioned, perfectly complementary siRNA-mediated cleavage at position 10 is probably the result of endonucleolytic cleavage by $\mathrm{AGO} 2$, the only AGO family member that is cleavage competent. Canonical cleavage products were cloned when imperfectly complementary siRNAs were transfected, suggesting that cleavage at position 10 might be the primary mechanism for downregulating off-target mRNAs. To test this hypothesis, we knocked down AGO2 in cells stably expressing one of the Renilla luciferase reporter constructs and measured RNA levels after transfection with an imperfectly complementary siRNA as previously described.

For these experiments, the cell line that stably expresses a Renilla luciferase reporter construct with a UAC trinucleotide at positions 9-11 was utilized (Table 1, mRNA reporter in column 3). Two CXCR4 siRNAs were used 
following AGO2 knockdown: one with perfect complementarity to the target sequence (Table 1, combination 27) and another with two mismatches at positions 10 and 11 (Table 1, combination 15). Cells were first transfected with Ago2 siRNAs and then transfected again with a CXCR4 siRNA $16 \mathrm{~h}$ later. The cells were collected $48 \mathrm{~h}$ after the Ago 2 siRNA transfection. As a control, GFP siRNA was also transfected on the same day as Ago2 siRNAs to assess the effect of an unrelated siRNA transfection on Renilla luciferase mRNA levels.

The extent of mRNA knockdown due to the perfectly and imperfectly complementary CXCR4 siRNAs was not significantly affected by the GFP siRNA transfection (Fig. 3A). mRNA knockdown due to the perfectly complementary CXCR4 siRNA decreased twofold when AGO2 was also

A

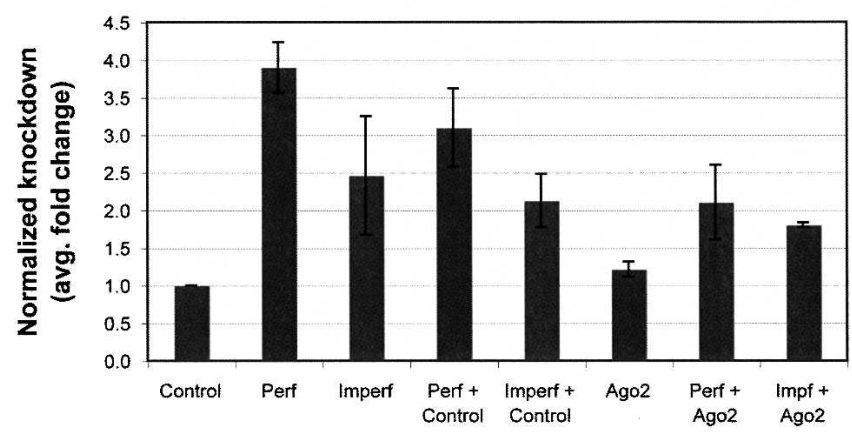

B

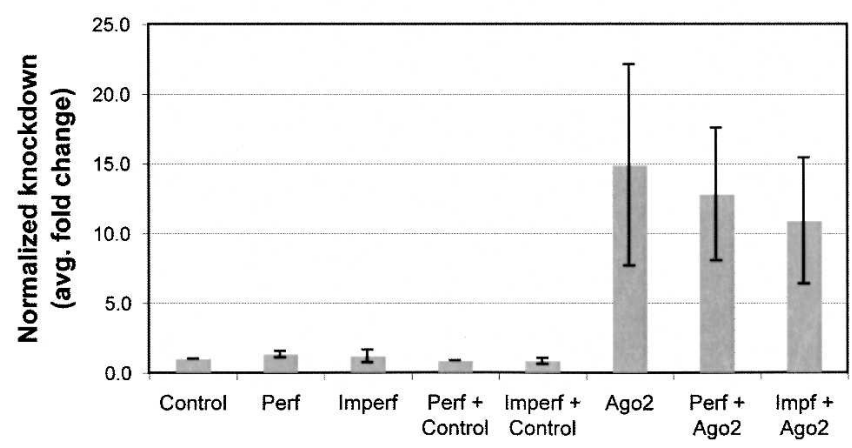

FIGURE 3. AGO2 is not required for off-target mRNA downregulation. (A) RNA analysis of a stably expressed Renilla luciferase reporter after transfection of $10 \mathrm{nM}$ of a perfectly complementary siRNA, CXCR4-D siRNA (combination 27-Perf), or $10 \mathrm{nM}$ of an imperfectly complementary siRNA, CXCR4-B (combination 15, mismatches at position 10 and 11-Imperf). These cells were transfected with either $100 \mathrm{nM}$ of an unrelated siRNA (targeting GFP) or $100 \mathrm{nM}$ of Ago2 siRNAs (Ago2-1 and Ago2-2) $16 \mathrm{~h}$ prior to the CXCR4 siRNAs transfections. (B) RNA analysis of Ago2 levels for experiment in $A$. RNA of analysis for $A$ and $B$ was performed by quantitative realtime PCR. TBP was used as an internal control. Samples were normalized to an unrelated siRNA (targeting GFP) transfection. Normalized average mRNA knockdown (fold change $\pm S D$ ) was calculated from two independent experiments. knocked down, whereas, mRNA knockdown due to transfection of the imperfectly complementary siRNA changed less than twofold. Ago2 mRNA levels were reduced 13- and 11-fold in cells transfected with perfectly and imperfectly complementary siRNAs, respectively (Fig. 3B). Given that the same degree of AGO2 knockdown was observed for cells transfected with perfectly or imperfectly complementary siRNA, insufficient AGO2 knockdown is not a likely explanation for why the mRNA reporter levels were not significantly changed for the imperfectly complementary mRNA:siRNA combination. These results indicate that AGO2-mediated cleavage and/or other functions performed by AGO2 are not solely responsible for off-target mRNA reductions and that other processes are probably involved in destabilizing off-target mRNAs.

\section{DISCUSSION}

Imperfectly complementary siRNAs frequently induce a moderate mRNA reduction. When this effect is observed in the context of the targeting of a specific mRNA by a perfectly complementary siRNA, these mRNA reductions are considered off-target effects. This effect may also be typical of mRNA:miRNA interactions.

The contribution of off-target effects to the phenotypes that result from siRNA targeted gene knockdown experiments is not well understood. There are several reports of unanticipated phenotypes generated by a siRNA to a specific mRNA, with off-target effects implicated in some cases (Scacheri et al. 2004; Lin et al. 2005; Fedorov et al. 2006). A recent study that examined the effects of siRNAs on cell physiology observed that 29\% (51/176) of a large siRNA population targeting two nonessential transcripts led to a reduction in cell viability of over 25\% (Fedorov et al. 2006). This phenotype mostly correlated with the presence of a UGGC motif within the siRNA guide strand. However, the general finding that expression of siRNAs from integrated short hairpin RNAs (shRNAs) can result in silencing of the target gene with little detectible effect on cell viability suggests that detrimental off-target effects are not common. Only one of the siRNAs utilized in this study contain the UGGC motif and none caused a reduction in cell viability. Therefore, the effects observed at the RNA and protein level in this study are likely mediated by partial complementarity to the siRNAs utilized and are unrelated to cell toxicity.

Published studies of off-target effects have focused largely on evaluating off-target mRNA regulation (Chi et al. 2003; Jackson et al. 2003; Semizarov et al. 2003; Persengiev et al. 2004; Birmingham et al. 2006). The effects of off-target regulation at the protein level have been characterized for only a few siRNA:mRNA combinations (Saxena et al. 2003; Scacheri et al. 2004; Jackson et al. 2006). In one of these studies, the degree of protein knockdown correlated with the previously observed changes 
in mRNA levels (Jackson et al. 2006). In two other studies, off-target gene regulation was largely associated with changes in protein levels and with less than twofold changes in RNA (Saxena et al. 2003; Scacheri et al. 2004). This study is the first in which off-target changes in both protein and mRNA levels are systematically characterized for a large number of mRNA:siRNA interactions.

Imperfectly complementary siRNAs can cause a spectrum of actions. At one end of the spectrum, we observe certain imperfectly complementary interactions that yield mRNA degradation with little additional translational repression. Conversely, certain mismatches result in significant protein reduction with little or no change in mRNA levels. Other mRNA:siRNA interactions fall in the middle of the spectrum, yielding some degree of mRNA degradation and translational repression. These results indicate that off-target activity can include regulation at the protein level that is independent of mRNA downregulation. Indeed, seven of the 30 siRNA:miRNA combinations studied here exhibited strong changes in protein levels and little or no change in mRNA levels. Two combinations (4 and 6) in this group had exact complementarity except for a G:U wobble in either position 9 or 10 . The other five examples (combinations 2, 10, 16, 22, and 28) corresponded to mRNA targets with only pyrimidines in positions 9-11.

A similar spectrum of mRNA and protein effects has recently been observed following transfection of a siRNA corresponding to a muscle-cell-specific miRNA, miR-1. Using stable isotope labeling with amino acids in cell culture (SILAC), Vinther and colleagues investigated the effects of transfecting miR-1 into HeLa cells on protein expression and compared these changes with previously identified mRNA changes determined by microarray analysis (Lim et al. 2005; Vinther et al. 2006). There was a slight overlap between the genes repressed by miR-1 at the mRNA and protein levels. In fact, four targets were identified for which corresponding protein and RNA repressions were observed. This report only identified 12 proteins that were repressed by miR-1 transfection.

Our detailed analysis of the different mismatch types tested revealed that G:U wobbles in positions 9 and 10, unlike other types of mismatches, resulted in significant protein knockdown with only modest changes at the mRNA level. Others have reported a loss in silencing at both the protein and mRNA levels with a $\mathrm{G}: \mathrm{U}$ wobble at position 10 (Holen et al. 2005). However, in agreement with our results, Saxena and colleagues have observed that $\mathrm{G}: \mathrm{U}$ wobbles in the central region of the anti-sense siRNA results in protein knockdown, similar to the degree of protein knockdown observed for perfectly complementary siRNAs, with few changes in mRNA levels (Saxena et al. 2003). This effect could potentially be explained if a G:U wobble in positions 9 and 10 caused a perturbation in the helix minor grove of the mRNA:siRNA duplex, leading to inhibition of endonucleolytic cleavage, but not binding.
Consistent with this hypothesis, Haley and Zamore have reported that base pairs formed by the central region of the siRNA guide strand contribute to formation of a A-form helix required for mRNA cleavage, but are not necessary for siRNA binding (Haley and Zamore 2004).

Mapping of endogenous CXCR4 degradation products generated after treatment with various imperfectly complementary siRNAs suggests that a siRNA-directed AGO2 cleavage event probably contributes to a fraction of the modest mRNA reduction we observed. Twenty-six percent $(10 / 39)$ and $14 \%(4 / 28)$ of the degradation products generated by the imperfectly complementary siRNAs, CXCR-A and CXCR4-B, respectively, mapped to position 10. In contrast to our results, other studies have not found that imperfectly complementary siRNA or miRNA binding results in the generation of canonical cleavage products. Degradation products of the C. elegans let-7 mRNA target, lin-41, mapped outside of the two let-7 binding sites (Bagga et al. 2005). Similarly, 5' RACE experiments performed on a luciferase reporter construct containing the $3^{\prime}$ UTR of the mammalian miR-125b target, lin-28, did not yield degradation products that mapped within the miR-125b binding sites ( $\mathrm{Wu}$ et al. 2006). A recent study observed that most of the degradation products recovered from a luciferase reporter containing three $3^{\prime}$ UTR let-7 binding sites mapped within the actual let-7 binding sites; however, none of the degradation products corresponded to the canonical cleavage site at position 10 (Schmitter et al. 2006). Compared to prior studies, it is possible that the higher degree of complementarity between our mRNA and siRNAs contributed to our detection of cleavage at the canonical position 10. Simultaneous knockdown of XRN1, which stabilizes 3' cleavage products, may also have contributed to our detection of canonical cleavage.

If reductions in mRNA levels were primarily due to canonical cleavage, then AGO2 inhibition should suppress the mRNA reductions observed with partially complementary siRNAs. However, we observed only a minor suppression of mRNA changes when AGO2 was knocked down in these cases. Thus, we conclude that an AGO2-independent pathway is also important in mediating downregulation of mRNA levels. Schmitter and colleagues have recently reported similar results, demonstrating that degradation of a luciferase reporter containing three $3^{\prime}$ UTR let-7 binding sites in mammalian cells only partially decreases when AGO2 is concomitantly knocked down (Schmitter et al. 2006). Another strong indication of an AGO2independent pathway for off-target mRNA degradation in our results is the mapping of numerous noncanonical 5' RACE products to sequences spanning the siRNA binding site. Similar products were very rare in cells transfected with control siRNAs. These products are probably generated by processes that are stimulated by the binding of a siRNA in an AGO2-independent fashion. Recently, various reports 
have implicated zebrafish miR-430 and mammalian let-7 and miR-125b in promoting deadenylation of target transcripts and subsequent mRNA decay (Giraldez et al. 2006; $\mathrm{Wu}$ et al. 2006). It appears likely that this pathway or another pathway yet to be defined is the AGO2-independent pathway responsible for a large majority of the mRNA changes observed with off-target interactions.

\section{MATERIAL AND METHODS}

\section{Construction of stable cell lines}

A modified version of the pRL-TK vector containing a multiplecloning site downstream of the Renilla luciferase stop codon was generated (pRL-TK $3^{\prime} \mathrm{MCS}$ ). To create stable cell lines that expressed different Renilla luciferase reporters, a pRL-TK $3^{\prime} \mathrm{MCS}$ $\mathrm{Bgl} \mathrm{II} / \mathrm{HpaI}$ fragment containing the Renilla luciferase gene and the SV40 poly(A) signal was cloned into a pPur BamHI/SwaI fragment containing the puromycin resistance gene (pRL-TK 3'MCS Pur). 3' UTR binding sites were constructed by annealing two DNA oligonucleotides containing two identical CXCR4 binding sites separated by the sequence CCGG and flanked by XhoI and SpeI sites. Annealed oligos were then inserted into pRLTK 3'MCS Puro's XhoI and SpeI sites, which are located directly downstream from the Renilla luciferase coding region. HeLa cells were then transfected with the modified pRL-TK 3'MCS Puro constructs containing different versions of the CXCR4 binding sites and selected with puromycin $(600 \mathrm{ng} / \mathrm{mL})$.

\section{SiRNAs}

siRNAs were purchased from Dharmacon and prepared according to manufacturer's instructions. Sequences of siRNAs (sense strand) used in this study are as follows:

CXCR4: 5'-GUUUUCACUCCAGCUAACAdTdT-3' CXCR4-A: 5'-GUUUUCACUGCAGCUAACAdTdT-3' CXCR4-B: 5'-GUUUUCACGCCAGCUAACAdTdT-3' CXCR4-C: 5'-GUUUUCACUCGAGCUAACAdTdT-3' CXCR4-D: 5'-GUUUUCACUACAGCUAACAdTdT-3' GFP: 5'-GGCUACGUCCAGGAGCGCAdTdT-3'

Firefly luciferase: $5^{\prime}$-CUUACGCUGAGUACUUCGAdTdT

Ago2-1: 5'-GCACGGAAGUCCAUCUGAAUU-3'

Ago2-2: 5' - GCAGGACAAAGAUGUAUUAUU-3'

Xrn1-beta: 5'-GUACCUGGAUAUACUAAGAdTdT-3'

Xrn1-delta: 5'-CUACUCAAGUACCUACUAAdTdT-3'

\section{Cell culture and transfections}

HeLa cells were grown in DMEM supplemented with 5\% inactivated fetal bovine serum, $5 \%$ calf serum, L-glutamine, and penicillin/streptomycin. The day before transfection, cells were seeded at $1.5 \times 10^{5}$ cells/well for six-well plates and $3.0 \times 10^{4}$ cells/well for 24-well plates in antibiotic-free media. Transfections were performed using Oligofectamine according to the manufacturer's instructions (Invitrogen) in a final volume of $1.250 \mathrm{~mL}$ for six-well plates and $0.25 \mathrm{~mL}$ for 24 -well plates. siRNAs were used at a final concentration of $5 \mathrm{nM}$ except when indicated otherwise.

\section{Real-time RT-PCR}

For detection of Renilla mRNA, total RNA was harvested $48 \mathrm{~h}$ after transfection using the RNAeasy kit (Qiagen) and DNAsetreated with RNA Free DNAse Set (Qiagen). Four micrograms of RNA were used for reverse transcription reactions with specific primers for Renilla luciferase (Rluc) and TATA binding protein (TBP), which served as an internal control. The following are the reverse transcription primers for TBP and Rluc: TBP RT primer 5'-GTACATGAGAGCCATTACGTCGTC-3'; Rluc RT primer $5^{\prime}$-GCATTCTAGTTGTGGTTTGTCC- ${ }^{\prime}$. The Rluc RT primer is located downstream of the $3^{\prime}$ UTR CXCR4 binding sites. Reverse transcription was performed using EndoFree RT (Ambion) according to manufacturer's guidelines. Resulting cDNA was diluted $1 / 5$ or $1 / 10$ and either 1 or $4 \mu \mathrm{L}$ of diluted cDNA were subjected to real-time PCR. Each PCR reaction was measured in quadruplicate. Rluc primers and probe were designed against the cDNA junction between pRL-TK vector sequence and the Renilla luciferase exon (pRL-TK contains a chimeric intron upstream of the Renilla reporter gene). The following are the primers and Taqman probes used for amplification of Rluc and TBP: Rluc forward primer (FP) 5'-TGCAGAAGTTGGTCGTGAGGCA-3'; Rluc reverse primer (RP) 5'-TCTAGCCTTAAGAGCTGTAATT GAACTGGG-3'; Rluc probe 5'-FAM-TGGGCAGGTGTCCACMGB-NFQ-3' (FAM: 6-carboxy-fluorescin; MGB: minor groove binding; NFQ: nonfluorescent quencher; Applied Biosystems); TBP primers and probe were obtained from Applied Biosystems (Taqman Gene Expression Assays, \# Hs00427620_m1). Real-time PCR was performed in a total volume of $25 \mu \mathrm{L}$, using the Taqman Universal PCR Master Mix protocol according to the manufacturer's instructions. Final concentrations of probes and primers used per reaction were $250 \mathrm{nM}$ and $900 \mathrm{nM}$, respectively. Increasing concentrations of cDNA were utilized to determine the linear range of real-time PCR. Titration experiments with five increasing concentrations of cDNA revealed that the TBP and Rluc Taqman probes and primers resulted in Ct values that were in the linear range. TBP and Rluc Taqman probe and primers both demonstrated a linear correlation with an $R^{2}$ value of 0.995 and 0.992, respectively. Subsequent experiments that utilized both TBP and Rluc Taqman probe/primers were performed with cDNA concentrations that were within the linear range established by the titration experiments. Relative mRNA levels were determined using the $2^{-\Delta \Delta \mathrm{Ct}}$ method. Control experiments measuring the change in $\Delta \mathrm{Ct}$ with different template dilutions showed similar efficiencies of amplification for the Renilla luciferase and TBP Taqman probes/primers. mRNA amounts were normalized to GFP siRNA transfections. For detection of Ago 2 and Xrn1 mRNA levels, total RNA was harvested $48 \mathrm{~h}$ after transfection as previously described. Two hundred fifty nanograms of RNA were utilized for reverse transcription reactions with oligo(dT). Reverse transcription was performed as previously described. One microliter of resulting CDNA was subjected to real-time PCR using Syber Green primers (SYBR). Each PCR reaction was measured in quadruplicate. The following are the primers used for amplification of Ago2, Xrn1, and TBP, which served as an internal control: Ago2 FP 5'-CGCGTCCGAAGGCTGCTCTA-3'; Ago2 RP 5'-TGGCTGTGCCTTGTAAAACGCT-3'; Xrn1 FP 5'-AGCTT TCGACTCCCGTTTCTCCAA-3'; Xrn1 RP 5'-GGCCAACACA TATCCTGGGTTGTA-3'; TBP FP 5'-GGAGAGTTCTGGGATTG TAC-3'; TBP RP 5'-CTTATCCTCATGATTACCGCAG-3'. Real 
time was performed in a total volume of $20 \mu \mathrm{L}$, using the SYBR Universal PCR Master Mix protocol according to the manufacturer's instructions. Relative mRNA levels were determined as previously described. Real-time reactions were conducted in an ABI PRISM 7000 Sequence Detection System Thermal Cycler (Applied Biosystems).

\section{Luciferase assays}

Renilla luciferase assays were performed $48 \mathrm{~h}$ after transfection according to the manufacturer's instructions (Promega) and detected with an Optocomp I luminometer (MGM Instruments). All of the readings obtained from luciferase assays were within the linear range (seven orders of magnitude) established by both the manufacturer (Promega) and by titration experiments previously performed in our laboratory.

\section{5' RACE}

HeLa cells were transfected with $100 \mathrm{nM}$ of Xrn1 siRNAs (Xrn1beta and Xrn1-delta) and $5 \mathrm{nM}$ of unrelated siRNAs (targeting GFP or firefly luciferase) or $5 \mathrm{nM}$ of perfectly and imperfectly complementary CXCR4 siRNAs. Total RNA was harvested $48 \mathrm{~h}$ after transfection using the RNAeasy kit (Qiagen) and DNAsetreated with RNA Free DNAse Set (Qiagen). Four micrograms of RNA were ligated to a synthetic RNA oligonucleotide adaptor (GCUGAUGGCGAUGAAUGAACACUGCGUUUGCUGGCUUU GAUGAAA) by treatment with T4 RNA ligase (2 units, New England Biolabs) for $1 \mathrm{~h}$ at $37^{\circ} \mathrm{C}$. After the ligation reaction, RNA was phenol/chloroform-extracted and precipitated with ethanol. Ligation products were reverse-transcribed using oligo(dT) primer and EndoFree RT (Ambion) according to the manufacturer's guidelines. The resulting cDNAs were amplified by successive rounds of PCR with a nested pair of primers. The first round of PCR was performed for 20 cycles using the following primers: RNA adaptor outer FP 5 '-GCTGATGGCGATGAATGA ACA-3' and CXCR4 outer RP 5' -CCTGCCTAGACACACATCA-3'. The second round of PCR was performed for 35 cycles using the following primers: RNA adaptor inner FP 5'-ACACTGCG TTTGCTGGCTTTGATG-3' and CXCR4 inner RP 5'-GAGACA TACAGCAACTAAGAACT-3'. RT-PCR products were examined by electrophoresis, gel purified, and cloned into pCR2.1TOPO vector for sequencing (Invitrogen).

\section{ACKNOWLEDGMENTS}

We thank T. Berzin, C. Cheng, and A. Leung for discussion and critical reading of the manuscript. We thank F. Solomon for critical scientific discussions. We thank C. Nielsen and C. Burge for sharing unpublished results. L.M.A. is a National Institutes of Health graduate fellow (5F31GM068983-03). This work was supported by United States Public Health Service RO1-GM34277 from the National Institutes of Health, U19 AI056900 from the National Cancer Institute to P.A.S., and partially by Cancer Center Support (core) grant P30-CA14051 from the National Cancer Institute.

Received October 17, 2006; accepted November 30, 2006.

\section{REFERENCES}

Bagga, S., Bracht, J., Hunter, S., Massirer, K., Holtz, J., Eachus, R., and Pasquinelli, A.E. 2005. Regulation by let-7 and lin-4 miRNAs results in target mRNA degradation. Cell 122: 553-563.

Behm-Ansmant, I., Rehwinkel, J., Doerks, T., Stark, A., Bork, P., and Izaurralde, E. 2006. mRNA degradation by miRNAs and GW182 requires both CCR4:NOT deadenylase and DCP1:DCP2 decapping complexes. Genes \& Dev. 20: 1885-1898.

Birmingham, A., Anderson, E.M., Reynolds, A., Ilsley-Tyree, D., Leake, D., Fedorov, Y., Baskerville, S., Maksimova, E., Robinson, K., Karpilow, J., et al. 2006. 3' UTR seed matches, but not overall identity, are associated with RNAi off-targets. Nat. Methods 3: 199-204.

Brennecke, J., Hipfner, D.R., Stark, A., Russell, R.B., and Cohen, S.M. 2003. bantam encodes a developmentally regulated microRNA that controls cell proliferation and regulates the proapoptotic gene hid in Drosophila. Cell 113: 25-36.

Brennecke, J., Stark, A., Russell, R.B., and Cohen, S.M. 2005. Principles of microRNA-target recognition. PLoS Biol. 3: e85.

Bronstein, I., Fortin, J., Stanley, P.E., Stewart, G.S., and Kricka, L.J. 1994. Chemiluminescent and bioluminescent reporter gene assays. Anal. Biochem. 219: 169-181.

Chi, J.T., Chang, H.Y., Wang, N.N., Chang, D.S., Dunphy, N., and Brown, P.O. 2003. Genomewide view of gene silencing by small interfering RNAs. Proc. Natl. Acad. Sci. 100: 6343-6346.

Ding, H., Schwarz, D.S., Keene, A., Affar el, B., Fenton, L., Xia, X., Shi, Y., Zamore, P.D., and Xu, Z. 2003. Selective silencing by RNAi of a dominant allele that causes amyotrophic lateral sclerosis. Aging Cell 2: 209-217.

Doench, J.G. and Sharp, P.A. 2004. Specificity of microRNA target selection in translational repression. Genes \& Dev. 18: 504-511.

Doench, J.G., Petersen, C.P., and Sharp, P.A. 2003. siRNAs can function as miRNAs. Genes \& Dev. 17: 438-442.

Fedorov, Y., Anderson, E.M., Birmingham, A., Reynolds, A., Karpilow, J., Robinson, K., Leake, D., Marshall, W.S., and Khvorova, A. 2006. Off-target effects by siRNA can induce toxic phenotype. RNA 12: 1188-1196.

Gazzani, S., Lawrenson, T., Woodward, C., Headon, D., and Sablowski, R. 2004. A link between mRNA turnover and RNA interference in Arabidopsis. Science 306: 1046-1048.

Giraldez, A.J., Mishima, Y., Rihel, J., Grocock, R.J., Van Dongen, S., Inoue, K., Enright, A.J., and Schier, A.F. 2006. Zebrafish MiR-430 promotes deadenylation and clearance of maternal mRNAs. Science 312: 75-79.

Haley, B. and Zamore, P.D. 2004. Kinetic analysis of the RNAi enzyme complex. Nat. Struct. Mol. Biol. 11: 599-606.

Holen, T., Moe, S.E., Sorbo, J.G., Meza, T.J., Ottersen, O.P., and Klungland, A. 2005. Tolerated wobble mutations in siRNAs decrease specificity, but can enhance activity in vivo. Nucleic Acids Res. 33: 4704-4710.

Jackson, A.L., Bartz, S.R., Schelter, J., Kobayashi, S.V., Burchard, J., Mao, M., Li, B., Cavet, G., and Linsley, P.S. 2003. Expression profiling reveals off-target gene regulation by RNAi. Nat. Biotechnol. 21: 635-637.

Jackson, A.L., Burchard, J., Schelter, J., Chau, B.N., Cleary, M., Lim, L., and Linsley, P.S. 2006. Widespread siRNA "off-target" transcript silencing mediated by seed region sequence complementarity. RNA 12: 1179-1187.

Jakymiw, A., Lian, S., Eystathioy, T., Li, S., Satoh, M., Hamel, J.C., Fritzler, M.J., and Chan, E.K. 2005. Disruption of GW bodies impairs mammalian RNA interference. Nat. Cell Biol. 7: 1267-1274.

Jing, Q., Huang, S., Guth, S., Zarubin, T., Motoyama, A., Chen, J., Di Padova, F., Lin, S.C., Gram, H., and Han, J. 2005. Involvement of microRNA in AU-rich element-mediated mRNA instability. Cell 120: 623-634.

Kasschau, K.D., Xie, Z., Allen, E., Llave, C., Chapman, E.J., Krizan, K.A., and Carrington, J.C. 2003. P1/HC-Pro, a viral suppressor of RNA silencing, interferes with Arabidopsis development and miRNA unction. Dev. Cell 4: 205-217. 
Kloosterman, W.P., Wienholds, E., Ketting, R.F., and Plasterk, R.H. 2004. Substrate requirements for let-7 function in the developing zebrafish embryo. Nucleic Acids Res. 32: 6284-6291.

Lewis, B.P., Burge, C.B., and Bartel, D.P. 2005. Conserved seed pairing, often flanked by adenosines, indicates that thousands of human genes are microRNA targets. Cell 120: 15-20.

Lim, L.P., Lau, N.C., Garrett-Engele, P., Grimson, A., Schelter, J.M., Castle, J., Bartel, D.P., Linsley, P.S., and Johnson, J.M. 2005. Microarray analysis shows that some microRNAs downregulate large numbers of target mRNAs. Nature 433: 769-773.

Lin, X., Ruan, X., Anderson, M.G., McDowell, J.A., Kroeger, P.E., Fesik, S.W., and Shen, Y. 2005. siRNA-mediated off-target gene silencing triggered by a $7 \mathrm{nt}$ complementation. Nucleic Acids Res. 33: 4527-4535.

Liu, J., Carmell, M.A., Rivas, F.V., Marsden, C.G., Thomson, J.M., Song, J.J., Hammond, S.M., Joshua-Tor, L., and Hannon, G.J. 2004. Argonaute2 is the catalytic engine of mammalian RNAi. Science 305: 1437-1441.

Liu, J., Rivas, F.V., Wohlschlegel, J., Yates 3rd, J.R., Parker, R., and Hannon, G.J. 2005. A role for the P-body component GW182 in microRNA function. Nat. Cell Biol. 7: 1261-1266.

Martinez, J. and Tuschl, T. 2004. RISC is a 5 'phosphomonesterproducing RNA endonuclease. Genes Dev. 18: 975-980.

Martinez, J., Patkaniowska, A., Urlaub, H., Luhrmann, R., and Tuschl, T. 2002. Single-stranded antisense siRNAs guide target RNA cleavage in RNAi. Cell 110: 563-574.

Orban, T.I. and Izaurralde, E. 2005. Decay of mRNAs targeted by RISC requires XRN1, the Ski complex, and the exosome. RNA 11: 459-469.

Persengiev, S.P., Zhu, X., and Green, M.R. 2004. Nonspecific, concentration-dependent stimulation and repression of mammalian gene expression by small interfering RNAs (siRNAs). RNA 10: $12-18$.

Petersen, C.P., Bordeleau, M.E., Pelletier, J., and Sharp, P.A. 2006. Short RNAs repress translation after initiation in mammalian cells. Mol. Cell 21: 533-542.

Rehwinkel, J., Natalin, P., Stark, A., Brennecke, J., Cohen, S.M., and Izaurralde, E. 2006. Genome-wide analysis of mRNAs regulated by Drosha and Argonaute proteins in Drosophila melanogaster. Mol. Cell. Biol. 26: 2965-2975.
Saxena, S., Jonsson, Z.O., and Dutta, A. 2003. Small RNAs with imperfect match to endogenous mRNA repress translation. Implications for off-target activity of small inhibitory RNA in mammalian cells. J. Biol. Chem. 278: 44312-44319.

Scacheri, P.C., Rozenblatt-Rosen, O., Caplen, N.J., Wolfsberg, T.G., Umayam, L., Lee, J.C., Hughes, C.M., Shanmugam, K.S., Bhattacharjee, A., Meyerson, M., et al. 2004. Short interfering RNAs can induce unexpected and divergent changes in the levels of untargeted proteins in mammalian cells. Proc. Natl. Acad. Sci. 101: 1892-1897.

Schmitter, D., Filkowski, J., Sewer, A., Pillai, R.S., Oakeley, E.J., Zavolan, M., Svoboda, P., and Filipowicz, W. 2006. Effects of Dicer and Argonaute downregulation on mRNA levels in human HEK293 cells. Nucleic Acids Res. 34: 4801-4815.

Schwarz, D.S., Ding, H., Kennington, L., Moore, J.T., Schelter, J., Burchard, J., Linsley, P.S., Aronin, N., Xu, Z., and Zamore, P.D. 2006. Designing siRNA that distinguish between genes that differ by a single nucleotide. PLoS Genet. 2: e140.

Semizarov, D., Frost, L., Sarthy, A., Kroeger, P., Halbert, D.N., and Fesik, S.W. 2003. Specificity of short interfering RNA determined through gene expression signatures. Proc. Natl. Acad. Sci. 100: 6347-6352.

Sen, G.L. and Blau, H.M. 2005. Argonaute 2/RISC resides in sites of mammalian mRNA decay known as cytoplasmic bodies. Nat. Cell Biol. 7: 633-636.

Song, J.J., Smith, S.K., Hannon, G.J., and Joshua-Tor, L. 2004. Crystal structure of Argonaute and its implications for RISC slicer activity. Science 305: 1434-1437.

Souret, F.F., Kastenmayer, J.P., and Green, P.J. 2004. AtXRN4 degrades mRNA in Arabidopsis and its substrates include selected miRNA targets. Mol. Cell 15: 173-183.

Vinther, J., Hedegaard, M.M., Gardner, P.P., Andersen, J.S., and Arctander, P. 2006. Identification of miRNA targets with stable isotope labeling by amino acids in cell culture. Nucleic Acids Res. 34: e107.

$\mathrm{Wu}$, L. and Belasco, J.G. 2005. Micro-RNA regulation of the mammalian lin-28 gene during neuronal differentiation of embryonal carcinoma cells. Mol. Cell. Biol. 25: 9198-9208.

$\mathrm{Wu}$, L., Fan, J., and Belasco, J.G. 2006. MicroRNAs direct rapid deadenylation of mRNA. Proc. Natl. Acad. Sci. 103: 4034-4039. 

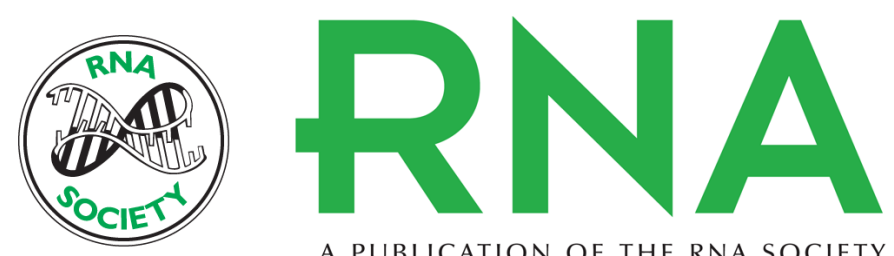

A PUBLICATION OF THE RNA SOCIETY

\section{Comparison of siRNA-induced off-target RNA and protein effects}

Lourdes M. Alemán, John Doench and Phillip A. Sharp

RNA 2007 13: 385-395 originally published online January 19, 2007

Access the most recent version at doi:10.1261/rna.352507

\section{References This article cites 43 articles, 19 of which can be accessed free at: http://rnajournal.cshlp.org/content/13/3/385.full.html\#ref-list-1}

\section{License}

Email Alerting Receive free email alerts when new articles cite this article - sign up in the box at the Service top right corner of the article or click here. 